\title{
Using Neutrinos as a Probe of the Strong Interaction
}

\author{
Jorge G. Morfín \\ Fermi National Accelerator Laboratory, P.O. Box 500, Batavia, IL, 60510
}

\begin{abstract}
Neutrino scattering experiments have been studying QCD for over 30 years. From the Gargamelle experiments in the early 70's, through the subsequent bubble chamber and electronic detector experiments in the 80 's and 90 's, neutrino scattering experiments have steadily accumulated increasing statistics and minimized their systematic errors. While the most recent study of QCD with neutrinos is from the TeVatron neutrino beam (the $\mathrm{NuTeV}$ experiment with results presented by Martin Tzanov at this Workshop), near-future studies will shift to the Main Injector based NuMI facility also at Fermilab. The NuMI Facility at Fermilab provides an extremely intense beam of neutrinos making it an ideal place for high statistics (anti)neutrino-nucleon/nucleus scattering experiments. The MINER $v$ A experiment at Fermilab is a collaboration of elementary-particle and nuclear physicists planning to use a fully active fine-grained solid scintillator detector to measure absolute exclusive cross-sections and nuclear effects in $v$ - A interactions as well as a systematic study of the resonance-DIS transition region and DIS with an emphasis on the extraction of high$\mathrm{x}_{B j}$ parton distribution functions. Further in the future an intense proton source, the Fermilab Proton Driver, will increase neutrino interaction rates by a further factor of 5 - 20 .
\end{abstract}

Keywords: <neutrino, scattering, nucleus>

PACS: <13.15>

\section{INTRODUCTION}

The results of the latest study of QCD using neutrino scattering comes from the $\mathrm{NuTeV}$ experiment [1]. The NuTeV experiment accumulated over 3 million $v$ and $\bar{v}$ events in the energy range of 20 to $400 \mathrm{GeV}$ and the details of the analysis can be found in Tzanov's contribution and contained references. The main points are that the $\mathrm{NuTeV}$ cross section agrees with the CCFR values (obtained using the same detector) for values of $\mathrm{x}_{B j} \leq 0.4$ but is systematically higher for larger values of $\mathrm{x}_{B j}$ culminating at $\mathrm{x}_{B j}=$ 0.65 where the NuTeV result is $20 \%$ higher than the CCFR result. NuTeV agrees with charged lepton data for $\mathrm{x}_{B j} \leq 0.5$ but there is increasing disagreement for higher values. Although $\mathrm{NuTeV} \mathrm{F}_{2}$ and $\mathrm{xF}_{3}$ agree with theory for medium $\mathrm{x}$, they find a different $\mathrm{Q}^{2}$ behavior at small $\mathrm{x}$ and are systematically higher than theory at high $\mathrm{x}$

These results can be summarized in four main questions to ask subsequent neutrino experiments:

- At low $x$, how does shadowing with incoming neutrinos differ from shadowing with incoming charged leptons?

- At high $\mathrm{x}$, what is the behavior of the valence quarks as $\mathrm{x} \rightarrow 1.0$ ? 
- At low $\mathrm{W}$, what is happening in the transition region between resonance production and the DIS regions?

- At all $\mathrm{x}$ and $\mathrm{Q}^{2}$, what is yet to be learned if we can measure all six $v$ and $\bar{v}$ structure functions to yield maximal information on the parton distribution functions?

At Fermilab, these questions will be addressed using the recently commissioned NuMI neutrino facility, designed for the MINOS neutrino oscillation experiment, and based on the Main Injector (MI) accelerator. The neutrino beams from the MI yield several orders of magnitude more events per $\mathrm{kg}$ of detector and year of running than the higher energy Tevatron neutrino beam. With such a facility, one can now perform statistically significant experiments with much lighter targets than the massive iron, marble and other high-A detector materials used in the past. That these facilities are designed to study neutrino oscillations points out the second advantage of NuMI neutrino experiments; an excellent knowledge of the neutrino beam will be required to reduce the beam-associated systematics of the oscillation result. This knowledge of the neutrino spectrum will also reduce the beam systematics in the measurement of neutrino scattering phenomena.

\section{THE MINER $v$ A NEUTRINO SCATTERING EXPERIMENT}

To take advantage of these major improvements, a collaboration of both elementary particle and nuclear physics institutions named MINERvA (Main Injector ExpeRiment: $v$ A) $[2,3]$ has been formed, received Stage I approval and is in the process of organizing funding with Fermilab's support.

The MINER $v$ A neutrino scattering experiment in the NuMI beam offers a unique opportunity to study a broad spectrum of physics topics. Several have not previously been studied in any systematic way, while others have only few results that are compromised by large statistical and systematic errors. A summary of these topics where MINERvA will break fresh ground include:

- Precision measurement of the quasi-elastic neutrino-nucleus cross-section, including its $E_{v}$ and $q^{2}$ dependence, and study of the nucleon axial form factors. Figure 1 shows the predictions for the cross section measurement assuming a 4-year MINERvA run. Figure 2 shows the extraction of the axial-vector form factor from the quasi-elastic event sample accumulated over a 4-year MINERvA run. The data points are plotted as a ratio of $F_{A} / F_{A}$ (Dipole). Also shown are the currently available values of $F_{A}$ from early experiments.

- Determination of single- and double-pion production cross-sections in the resonance production region for both neutral-current and charged-current interactions, including a study of isospin amplitudes, measurement of pion angular distributions, isolation of dominant form factors, and measurement of the effective axial-vector mass.

- Clarification of the W ( $\mathrm{W}$ mass of the hadronic system) transition region wherein resonance production merges with neutrino deep-inelastic scattering, including 




FIGURE 1. Cross section for MINER $v$ A assuming a 4 year run with $M_{A}=1.00 \mathrm{GeV}$, and the Fermi gas model.Statistical errors only are shown

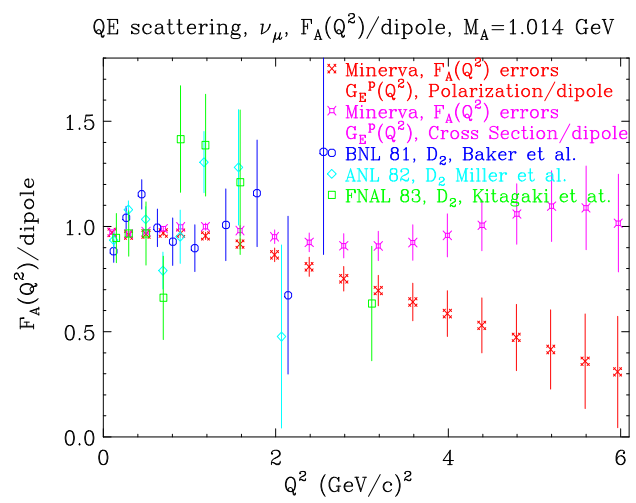

FIGURE 2. Extracted values of $F_{A}\left(q^{2}\right) /$ dipole for deuterium bubble chamber experiments Baker et al. [4] and Kitagaki et al. [5]. For MINER $v$ A the projected results are shown for two different assumptions: $F_{A} /$ dipole $=G_{E}^{p} /$ dipole from cross section and $F_{A} /$ dipole $=G_{E}^{p} /$ dipole from polarization. The MINER $v \mathrm{~A}$ errors are for a 4 year run.

tests of phenomenological characterizations of this transition such as quark/hadron duality.

- Precision measurement of coherent single-pion production cross-sections, with particular attention to target A dependence. Coherent $\pi^{0}$ production, via the neutralcurrent, is a significant background for next-generation neutrino oscillation experiments seeking to observe $v_{\mu} \rightarrow v_{e}$ oscillation. Figure 3 shows the expected precision (statistical errors only) of the MINER $v$ A measurement of the charged current coherent pion production cross-section as a function of neutrino energy. Here it is assumed that the measured value is that predicted by Rein-Seghal. Also plotted are the only currently available measurements showing their total errors. Figure 4 illustrates the broad range in A to be covered by MINERvA's measurement of the 


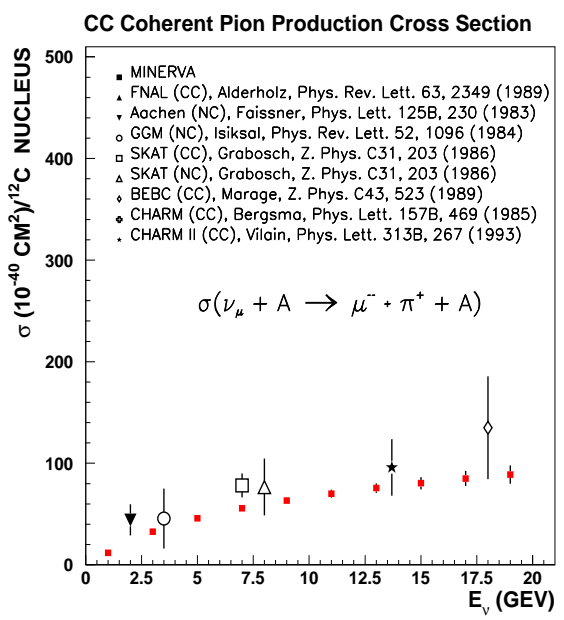

FIGURE 3. Coherent cross-sections as measured by MINERvA compared with existing published results. MINERvA errors here are statistical only.

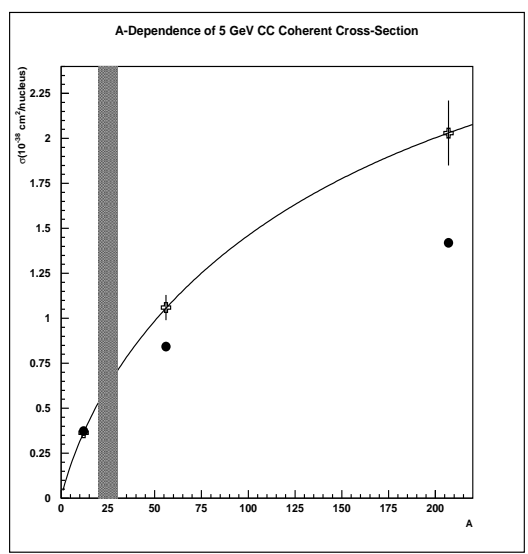

FIGURE 4. The range of A-dependent measurements of the coherent-pion cross-section to be measured by the MINER $v$ A experiment

coherent pion cross-section. The shaded band is the range in A covered by existing experiments.

- Examination of nuclear effects in neutrino-induced interactions including energy loss and final-state modifications in heavy nuclei. These nuclear effects play a significant role in neutrino oscillation experiments that measure $v_{\mu}$ disappearance as a function of $E_{v}$. With sufficient $\bar{v}$ running, a study of quark flavor-dependent nuclear effects will also be performed.

- Clarification of the role of nuclear effects as they influence the determination of 
$\sin ^{2} \theta_{W}$ via measurement of the ratio of neutral-current to charged-current crosssections off different nuclei.

- Much-improved measurement of the parton distribution functions will be possible using a measurement of all six $v$ and $\bar{v}$ structure functions (with sufficient $\bar{v}$ running).

- Examination of the leading exponential contributions of perturbative QCD.

- Precision measurement of exclusive strange-particle production channels near threshold, thereby improving knowledge of backgrounds in nucleon-decay searches, determination of $V_{u s}$, and enabling searches for strangeness-changing neutral-currents and candidate pentaquark resonances. Measurement of hyperonproduction cross-sections, including hyperon polarization, is feasible with exposure of MINER $v$ A to $\bar{v}$ beams.

- Studies of nuclear physics for which neutrino reactions provide information complementary to JLab studies in the same kinematic range.

In addition to being significant fields of study in their own right, improved knowledge of many of these topics is essential to minimizing systematic uncertainties in neutrinooscillation experiments.

\subsection{The MINER $v$ A Detector}

For MINER $v$ A to meet its physics goals, the detector must break new ground in the design of high-rate neutrino experiments. With final states as varied as high-multiplicity deep-inelastic reactions, coherent single- $\pi^{0}$ production and quasi-elastic neutrino scattering, the detector is a hybrid of a fully-active fine-grained detector and a traditional calorimeter. A complete description of MINERvA is found in the proposal [3]. The essential features are described here.

The MINER $v$ A detector is made up of a number of sub-detectors with distinct functions in reconstructing neutrino interactions. The fiducial volume for most analyses is the inner "Active Target" shown in Figure 5, where all the material of the detector is the scintillator strips themselves. The scintillator detector does not fully contain events due to its low density and low $Z$, and therefore, the MINER $v A$ design surrounds the scintillator fiducial volume with sampling detectors. To construct these sampling detectors, the scintillator strips are intermixed with absorbers. For example, the side, upstream (US) and downstream (DS) electromagnetic calorimeters (ECALs) have lead foil absorbers. Surrounding the ECALs are the US and DS hadronic calorimeter (HCAL) where the absorbers are steel plates. The US ECAL and HCAL serve a dual purpose as high-A targets for the study of nuclear effects. On the side of the detector, it is the outer detector (OD) that plays the role of the HCAL. Upstream of the detector is a veto of steel and scintillator strips to shield MINER $v$ A from incoming soft particles produced upstream in the hall.

The core active element will be triangularly-shaped extruded scintillator strips readout via wavelength-shifting fibers, Readout of the fibers will be done with multi-anode photomultiplier tubes (MAPMTs), connected to the wavelength shifting fibers via an 


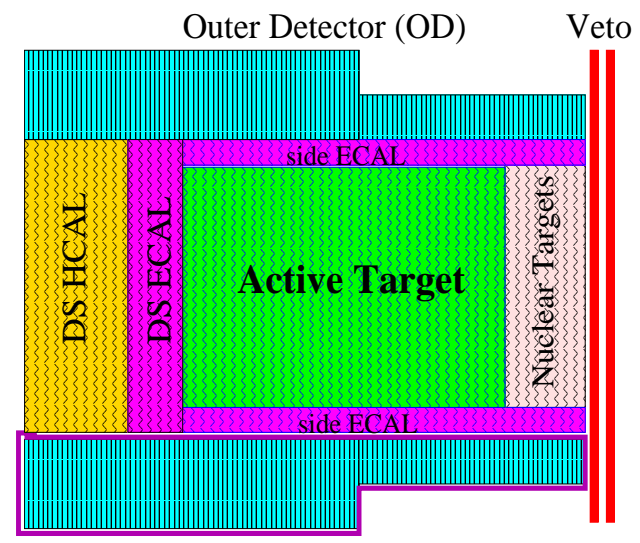

FIGURE 5. A side view schematic of the MINER $v$ A detector

optical cable system and housed in a light tight "optical box" mounted on the outer detector. There are three distinct orientations of strips in the inner detector and veto, separated by $60^{\circ}$, and labeled X, U, V. A single module of MINER $v$ A has two X layers to seed two-dimensional track reconstruction, and one each of the $\mathrm{U}$ and $\mathrm{V}$ layers to reconstruct three-dimensional tracks.

The MINER $v$ A detector will be placed directly upstream of the MINOS near detector. Forward going muons exiting MINERvA can be measured in the MINOS near detector either by range or curvature.

\section{A FUTURE NEUTRINO DETECTOR FOR NUMI}

In a subsequent stage it would be most beneficial, for all physics topics of interest, to have a low-A target, preferably $\mathrm{H}_{2}$ or $D_{2}$. An investigation of the technical and safety challenges of such a target is currently underway at Fermilab and a report [7] indicates that there are no real technical challenges in fabricating or efficiently operating a large $\mathrm{LH}_{2}$ or $\mathrm{L} D_{2}$ target. The main effort (and expense) for such a facility would be in satisfying safety requirements. For a fiducial volume with $\mathrm{r}=80 \mathrm{~cm}$. and $1=150 \mathrm{~cm}$. we would expect $560 \mathrm{~K} \mathrm{CC}$ events in $\mathrm{LH}_{2}$ and $1280 \mathrm{~K} \mathrm{CC}$ events in $\mathrm{L}_{2}$ per year of he- $v$ running.

An alternative proposal for a $\mathrm{H}_{2}$ or $\mathrm{D}_{2}$ target suggests that modern bubble chamber techniques, including recently-developed exceptionally smooth walls to eliminate extraneous bubbles and the use of CCD readout to allow electronic automatic scanning of events, makes a fully active liquid $H_{2}$ or $D_{2}$ target quite reasonable.

\section{THE FERMILAB PROTON DRIVER}

It has become increasingly clear that future long baseline neutrino experiments will require further factors of 5-10 improvements in proton luminosity. Such experiments 
at Fermilab are only feasible if a major proton source upgrade is undertaken. The Proton Driver project would replace the Booster with a new 8-GeV accelerator with 0.5-2 MW beam power, a factor of 15-60 more than the current Booster. It would also make the modifications needed to the Main Injector to upgrade it to simultaneously provide 120 $\mathrm{GeV}$ beams of $2 \mathrm{MW}$. A Proton Driver would bring with it other advantages. It would have the capacity to support a vigorous $8-\mathrm{GeV}$ fixed-target program while providing 2 MW Main Injector beams. A Fermilab report [8] summarizes the physics potential of such a program.

A Proton Driver can also serve as a stepping-stone to future accelerators, both as an R\&D test bed and as an injector, with connections to the Linear Collider, Neutrino Factories, and a VLHC.

A design study for a Superconducting Linac-based Proton Driver has been completed [9]. The simplicity of design should make it simpler to operate than booster/linac combinations. Limited emittance growth in a linac means that it can deliver the high brightness, low halo beams needed for running the Main Injector at high intensity with acceptable losses. The short MI "fill time" could deliver the full $2 \mathrm{MW}$ of beam power at any energy from 40 to $120 \mathrm{GeV}$, and improvements to the MI ramp time could further increase the average proton intensity. There are many technical overlaps between the development and construction of such a machine and a cold technology Linear Collider. The $8 \mathrm{GeV}$ superconducting linac could also accelerate electrons and could serve as a stepping-stone to future accelerators, both as an R\&D test bed and as an injector, with connections to the Linear Collider, Neutrino Factories, and a VLHC.

Such a proton driver would allow an $8 \mathrm{GeV}$ proton based neutrino program with a factor of $\approx 20$ increase in intensity compared to the current MiniBooNe experiment and a factor 5 increase in the $120 \mathrm{GeV}$ proton based Main Injector neutrino program compared to MINOS.

\section{REFERENCES}

1. M. Tzanov, 'NuTeV Structure Function Measurement”, in these Proceedings.

2. The MINERvA Collaboration consists of groups from the following institutions: $U$ Athens, $U$ California/Irvine, U Dortmund, Fermilab, Hampton U, IL Inst. Tech., Inst. for Nuc. Research Moscow, James Madison U, Jefferson Lab, N. Illinois U, U Pittsburgh, U Rochester, Rutgers U, Tufts U, William and Mary U.

3. D. Drakoulakos et al. [The MINERvA Collaboration], "Proposal to Perform a High-Statistics Neutrino Scattering Experiment Using a Fine-grained Detector in the NuMI beam”, December 2003 [rev. January 2004]. Submitted to the FNAL PAC. http: / / www . pas . rochester. edu/minerva/

4. N. J. Baker et al., Phys. Rev. D23, 2499 (1981).

5. T. Kitagaki et al., Phys. Rev. D26, 436 (1983).

6. P. Rubinov, FNAL-TM-2226. P. Rubinov, FNAL-TM-2227. P. Rubinov, FNAL-TM-2228.

7. J. Kilmer and T. J.Sarlina, "Technical and Safety Considerations of Large Cryogenic Liquid Targets at the NuMI Near Hall" contribution to the Workshop on New Initiatives for the NuMI Neutrino Beam, Fermilab, 2002

8. S. Geer, Nucl.Phys.Proc.Suppl. 147, 124-127 (2005)

9. R. D. Kephart,, Nucl.Phys.Proc.Suppl. 147, 41-44 (2005) 